\title{
Ayuda psicológica a cuidadores en diferentes escenarios
}

\author{
Andrés Losada ${ }^{1}$, Celia Nogales-González ${ }^{1}$, Javier López ${ }^{2}$, María Márquez-González $z^{3} y$ \\ Estefanía Jiménez-González ${ }^{1}$ \\ 'Departamento de Psicología, Universidad Rey Juan Carlos, Madrid \\ ${ }^{2}$ Departamento de Psicología, Universidad CEU San Pablo, Madrid \\ ${ }^{3}$ Departamento de Psicología Biológica y de la Salud, Universidad Autónoma de Madrid
}

\begin{abstract}
IOSUMOH/RhStract:
El cuidado familiar de una persona con demencia se asocia con importantes consecuencias negativas para la salud psicológica y física de los cuidadores. Si bien se ha utilizado el término "carrera" como metáfora que permite describir las diferentes etapas por la que suelen pasar las personas que cuidan, la realidad es que no es frecuente en la literatura científica que se realice una distinción sobre intervenciones específicas para las diferentes condiciones contextuales o situacionales en las que se encuentra un cuidador. En el presente trabajo se lleva a cabo un análisis de intervenciones específicas que pueden ayudar a los cuidadores en diferentes escenarios. Específicamente, se revisan intervenciones para entrenar a los cuidadores en habilidades para el cuidado y el autocuidado, intervenciones con cuidadores que faciliten y optimicen el uso de centros de día, intervenciones para familias que se encuentren en el proceso de institucionalización del familiar en contextos residenciales y, finalmente, se revisan las posibilidades de actuación psicológica para el afrontamiento del duelo de los cuidadores. Dado el importante número de cuidadores que hay (y habrá) en nuestro país, que contrasta con la escasez de recursos disponibles para cuidadores, parece importante destinar importantes esfuerzos y recursos al desarrollo, evaluación e implantación de intervenciones para cuidadores en los diferentes contextos en los que estos se pueden beneficiar de las mismas.
\end{abstract}

Dementia family caregiving is linked with significant negative consequences for caregivers' psychological and physical health. Even though the term "career" has been used as a metaphor that describes the different caregiving transitions, it is not frequent to find in the caregiving literature information about interventions that may be especially useful in specific caregiving contexts or situations. In this work we review interventions that may help caregivers in different settings. Specifically, we review interventions aimed at training caregivers in self-care strategies, interventions that may help caregivers to optimize day care use, interventions for family caregivers who are in the process of institutionalizing their relative or whose relative is institutionalized and, finally, interventions for bereaved caregivers facing the death of their relative. Considering the important percentage of population who are primary caregivers in our country, and the shortage or resources available to them, it seems very important to devote important efforts and resources to the development, assessment, and implementation of psychological interventions for caregivers, specifically targeted to the different situations and contexts they face.

\section{nalabras clave/keywords:}

Autocuidado, centro de día, cuidadores, demencia, duelo, intervención, psicológica, psicoterapia, residencias Self-care, daycare, caregivers, dementia, bereavement, intervention, psychological, psychotherapy, nursing home.

\section{Agradecimientos}

El presente trabajo se ha realizado en el marco del proyecto PSI2009-08132/PSIC, financiado por el Ministerio de Ciencia e Innovación, y del proyecto PSI2012-31293, financiado por el Ministerio de Economía y Competitividad. 


\section{1- Introducción}

La atención a las personas mayores dependientes se ha llevado a cabo tradicionalmente por las familias, especialmente por las mujeres, estimándose que el $83 \%$ de la atención que reciben las personas mayores dependientes es proporcionada en el contexto familiar (IMSERSO, 2005). Factores como el envejecimiento de la población y el aumento de la esperanza de vida, unidos a otros como la incorporación de la mujer al mundo laboral remunerado y la reducción de la natalidad, están provocando un aumento significativo del porcentaje de población dependiente y una reducción significativa del número y tamaño de las redes informales de apoyo. La relevancia del fenómeno de la dependencia ha favorecido actuaciones como la aprobación de la ley de promoción de la autonomía personal y atención a personas en situación de dependencia (BOE, 2006; Ley 39/2006). Además de que las expectativas generadas por la aplicación de esta ley no están siendo satisfechas, especialmente debido al contexto de crisis económica (Mateos, Franco y Sánchez, 2010), dicha legislación presenta importantes debilidades en cuanto a la atención a las personas cuidadoras tales como, por ejemplo, una escasa o nula referencia a la importancia de las intervenciones con cuidadores (p.ej., Losada, Márquez-González, Peñacoba, Gallagher-Thompson y Knight, 2007).

Cuidar de un familiar mayor con dependencia, especialmente si ésta es debida a una demencia, se asocia con importantes niveles de malestar psicológico y físico (Mausbach, Roepke, Ziegler, Milic, von Känel, Dimsdale, Mills, Patterson, Allison, Ancoli-Israely Grant, 2010; Pinquarty Sörensen, 2003) e incluso con riesgo de muerte, siendo la probabilidad de morir de un cuidador que manifiesta estrés un $63 \%$ superior a la de un no cuidador (Schulzy Beach, 1999). El cuidado de personas mayores con demencia se ha considerado una situación prototípica de estrés crónico (Vitaliano et al., 2004), siendo el modelo de estrés y afrontamiento el que principalmente ha guiado el análisis del malestar del cuidador, habiendo recibido abundante apoyo empírico hasta la fecha (ver. p.ej., Knight y Sayegh, 2010). Este modelo permite identificar variables que contribuyen a explicar por qué el cuidado impacta más o menos en la salud psicológica y física de los cuidadores. Así, por ejemplo, variables como el apoyo social o la autoeficacia parecen amortiguar significativamente el efecto negativo del cuidado sobre los cuidadores (Crespo, López y Zarit, 2005; RomeroMoreno, Losada, Mausbach, MárquezGonzález, Patterson, López, 2011). Por el contrario, variables como los pensamientos disfuncionales sobre el cuidado o la evitación experiencial, parecen contribuir negativamente al malestar del cuidador (Losada, Márquez-González, Knight, Yanguas, Sayegh y Romero-Moreno, 2010; Márquez-González, Romero-Moreno y Losada, 2010), al asociarse a pautas de afrontamiento del cuidado desadaptativas.

La consideración del cuidado como un estresor crónico tiene también que ver con la duración del proceso, habitualmente de años (Larson, Shadlen, Wang, McCormick, Bowen, Teri y Kukull, 2004). Esta circunstancia ha provocado que en ocasiones se utilice el término "carrera" como metáfora que permite describir las diferentes etapas por la que suelen pasar las personas que cuidan (ver, p.ej., Pearlin, Mullan, Semple y Skaff, 1990). Así, por ejemplo, los cui- 
dadores pasan inicialmente por un proceso de asunción de la tarea de cuidados. Los motivos por los que el cuidador asume el rol de cuidador pueden ser diversos, y han sido descritos como intrínsecos (por ej., cuido porque quiero) o extrínsecos (p.ej., cuido porque no me queda otra opción). Parece que aquellos cuidadores que cuidan por motivos intrínsecos se encuentran en mejores condiciones que aquellos que lo hacen por motivos meramente extrínsecos (Romero-Moreno, Losada, Mausbach, Márquez-González, Patterson y López, 2011). Durante las primeras fases de la enfermedad el cuidado del familiar se lleva, en la mayoría de los casos, en el entorno domiciliario. A lo largo de estas fases los cuidadores se enfrentan a desafíos como conocer en qué consiste la enfermedad, aprender a cuidar, o adaptarse a la nueva situación. Es muy frecuente que los cuidadores renuncien (explícita o implícitamente) a sus necesidades personales (relaciones sociales, laborales, familiares y personales, ocio, descanso, etc.) y se concentren exclusivamente en la atención y supervisión de su familiar (IMSERSO, 2005). También es frecuente que asuman en exclusiva la tarea de cuidar, e incluso que no se utilicen recursos formales, bien por desconocimiento de los mismos o bien por rechazo explícito, por influencia de variables socioculturales (Losada, Knight y Márquez, 2003). La utilización de recursos formales (por ej., centros de día) parece ser recomendable para los cuidadores (Gottlieb y Johnson, 2000), dado que les puede proporcionar un "respiro" en su tarea que, a su vez, les puede facilitar responder a otras demandas o necesidades (p.ej., autocuidado, familia, o trabajo).

$\mathrm{Si}$ bien en muchas ocasiones las personas que cuidan realizan su tarea hasta el final en el domicilio, algunos cuidadores toman la generalmente difícil decisión de ingresar a su familiar en una residencia. Para algunas familias no existe otra alternativa que la institucionalización, y el que ésta se produzca no significa que el proceso del cuidado termine. Al contrario, la mayoría de las familias desea mantener un rol activo en la atención a su familiar (Márquez-González, Losada, Pillemer, Romero-Moreno, López y Martínez, 2010). La asociación entre el ingreso del familiar en una residencia y el malestar del cuidador no está clara, existiendo resultados mixtos, que en ocasiones sugieren una reducción del malestar (Grant, Adler, Patterson, Dimsdale, Ziegler e Irwin, 2002), mientras que en otras sugieren que el malestar se mantiene, o incluso aumenta (Lieberman y Fisher, 2001; Schulz, Belle, Czaja, McGinnis, Stevens y Zhang, 2004).

Finalmente, aunque podría pensarse que el fallecimiento del familiar pone fin al proceso del cuidado, la realidad es que en muchos casos las consecuencias negativas asociadas al cuidado (tristeza, culpa, riesgo cardiovascular, etc.) se mantienen tras el fallecimiento, o incluso se observan fenómenos como el duelo anticipado (Robinson-Whelen, Tada, MacCallum, McGuire y Kiecolt-Glaser, 2001).

En la mayoría de los artículos en los que se ha revisado la eficacia de las intervenciones para cuidadores (por ej., Gallagher-Thompson, Tzuang, Brodaty, Charlesworth, Gupta, Lee, Losada y Shyu, 2012; Pinquart y Sörensen, 2006) no se ha hecho una distinción sobre intervenciones específicas para las diferentes condiciones contextuales $\mathrm{o}$ situacionales en las que se encuentra un cuidador. Sin embargo, las circunstancias antes comentadas parecen sugerir la con- 
veniencia de llevar a cabo un análisis de intervenciones específicas que pueden ayudar a los cuidadores en diferentes escenarios. Así, en el presente artículo se llevará a cabo una revisión de las intervenciones que pueden ayudar a los cuidadores en los siguientes escenarios: entrenamiento en habilidades para el cuidado y el autocuidado, intervenciones con cuidadores que faciliten y optimicen el uso de centros de día, atención a los familiares en el proceso de institucionalización en contextos residenciales $\mathrm{y}$, finalmente, se revisarán las posibilidades de actuación psicológica para el afrontamiento del duelo.

\section{2- Intervenciones psicoeducativas 0 psicoterapéuticas para cuidadores}

El impacto cada vez mayor que el cuidado de las personas mayores dependientes tiene sobre la sociedad se ve reflejado en el número de publicaciones que se vienen realizando sobre intervenciones para cuidadores (ver p. ej., Gallagher-Thompson et al., 2012). Si bien el diseño y los procedimientos de estas intervenciones han ido ganando en calidad, la realidad es que, en el momento actual, el tamaño del efecto de las intervenciones para reducir el malestar de los cuidadores es, en el mejor de los casos, moderado (p. ej., Pinquarty Sörensen, 2006). De los diferentes tipos de intervención existentes (p.ej., respiro, autoayuda, educación o psicoterapia), las intervenciones cognitivo-conductuales son las que muestran un mayor tamaño del efecto $\mathrm{y}$, dentro de éstas, las realizadas en formato individual obtienen mejores resultados que las realizadas en formato grupal (Gallagher-Thompson y Coon, 2007; Pinquart y Sörensen, 2006).

Las intervenciones psicoterapéuticas y psicoeducativas son, junto con las interven- ciones multicomponente (p.ej., Mittelman, Roth, Clay y Haley, 2007) y las de modificación de conducta (Logsdon, McCurry y Teri, 2007), las únicas que han recibido suficiente respaldo empírico como para ser calificadas como científicamente validadas (Gallagher-Thompson y Coon, 2007).

Se ha recomendado el desarrollo de intervenciones fundamentadas en modelos teóricos respaldados empíricamente como un procedimiento para aumentar la efectividad de las intervenciones, dado que permite establecer los mecanismos de acción a través de los cuáles se espera que se produzcan los cambios deseados, y los resultados que son esperables y sobre qué variables (Knight y Losada, 2011). Modelos teóricos como el de estrés y afrontamiento adaptado al cuidado (p.ej., Knighty Sayegh, 2010), el modelo cognitivo conductual adaptado al cuidado (Losada et al., 2003) o el modelo más reciente "acontecimientos placenterosrestricción de actividades" (modelo PEAR, Mausbach et al., 2011) son referencias fundamentales en este sentido. El desarrollo de procedimientos para atender a la fidelidad del tratamiento o implementación de la intervención también ha sido sugerido como vía para aumentar la eficacia de las intervenciones (Burgio, Corcoran, Lichstein, Nichols, Czaja, Gallagher-Thompson, Bourgeois, Stevens, Ory y Schulz, 2000; Zarit y Femia, 2008).

Zarit, Femia, Kim y Whitlatch (2010) señalan que las intervenciones deben estar dirigidas a objetivos específicos concretos y no a una problemática genérica supuestamente compartida por todos los cuidadores como grupo, tal y como se ha hecho hasta ahora. Así, si el objetivo de una intervención es reducir la sintomatología depresiva de los cuidadores, ésta: a) debería estar dirigida 
específicamente a cuidadores que manifiesten niveles significativos de depresión; y b) debería incluir contenidos específicamente diseñados para tratar la depresión, modificando las variables concretas implicadas en su mantenimiento. Las diferencias en los efectos psicológicos y físicos del cuidado en función de variables como el sexo del cuidador o el parentesco con la persona cuidada (Pinquart y Sörensen, 2011), y la evidente heterogeneidad de los cuidadores en cuanto a las situaciones que han de afrontar y sus necesidades de ayuda, recomiendan la potenciación de estudios que, o bien analicen la eficacia diferencial de las intervenciones en función de este tipo de variables (Pinquart y Sörensen, 2011), o bien planteen intervenciones ajustadas al perfil y necesidades de tratamiento específicas de cada cuidador (Zarit et al., 2010).

Con base en nuestra experiencia de intervención con cuidadores y en el análisis de la literatura psicológica, podemos añadir el problema del "encorsetamiento" y falta de flexibilidad que supone, en cierta medida, la aplicación guiada por un manual de un tratamiento, sin poder tener en cuenta las particularidades y necesidades específicas de cada caso individual. Sin dudar de la importancia y ventajas de este tipo de diseños, esta aproximación presenta importantes limitaciones. Por un lado, su énfasis en el control experimental de variables actúa en detrimento de su validez ecológica, perdiéndose muchos aspectos de la intervención clínica real. De hecho, en la realidad clínica la terapia no tiene duración ni estructura fija y es, además, autocorrectiva: si algo no va bien, se revisa el proceso y se introducen cambios (Seligman, 1995). La "manualización" de los tratamientos ha sido y continúa siendo un tema polémico.
Así, los tratamientos empíricamente validados serían en gran medida "artificiales" $y$, pudiendo ser muy eficaces, no son siempre tan efectivos, útiles y/o aplicables en el contexto clínico real, entre otras razones porque muchos terapeutas rechazan esa forma de hacer terapia que no se ajusta al caso individual (p.ej., Addis y Krasnow, 2000). Por otro lado, los tratamientos manualizados se han desarrollado como paquetes de intervención breve (generalmente unas ocho sesiones), en gran medida con base a criterios de parsimonia, eficiencia y facilitación del control experimental y la investigación, fundamentalmente asociados a la falta de tiempo de los cuidadores para participar en intervenciones prolongadas que exigen una dedicación semanal. Sin embargo, como se ha sugerido, atendiendo a criterios de efectividad (satisfacción de los cuidadores), debemos reconocer que los programas de intervención resultan excesivamente breves para muchos cuidadores.

Tras la revisión de diversos trabajos que hablan de intervenciones para cuidadores (p.ej., Gallagher-Thompson et al., 2012; Pinquart y Sörensen, 2006; Zarit y Femia, 2008) puede concluirse que las características de una intervención que parecen asociarse a un mayor éxito de las mismas para entrenar a los cuidadores en habilidades que les permitan un afrontamiento más adaptativo del cuidado, así como reducir su malestar, son: a) ser experto en el problema, conociendo los modelos teóricos y las intervenciones que han mostrado mayor eficacia hasta el momento; b) realizar una adecuada evaluación que permita identificar las áreas clave de necesidad de cada cuidador; c) llevar a cabo una intervención ajustada a las particularidades de cada caso (por este motivo una intervención indivi- 
dual parece ser más efectiva que una grupal), flexible en la medida en que se pueda ajustar a los posibles cambios que acontezcan en el proceso del cuidado; d) entrenar, y no solo educar, promoviendo una participación activa del cuidador en el proceso de la intervención; e) implicar a otros familiares en el proceso de la intervención y f) evaluar, además de los resultados de la intervención, el proceso de la misma o la implementación de la intervención.

\section{3- Atención a familias en centros de día (o centros de atención especializada a personas mayores)}

Desde los años 80 la investigación sobre el cuidado de las personas mayores ha encontrado un mayor equilibrio entre los estudios que evalúan la carga del cuidador familiar y los estudios que evalúan el impacto de los servicios e intervenciones diseñadas para disminuir los efectos estresantes del cuidado (Gottlieb y Johnson, 2000).

De entre estos servicios, los de respiro se encuentran entre los más deseados por los cuidadores y persiguen el objetivo de prevenir o posponer el ingreso residencial (Gottlieb y Johnson, 2000), siendo el más prevalente el uso de centros de día (Gottlieb y Johnson, 2000), (IMSERSO, 2008), uno de los pocos servicios que tiene como foco principal el mantenimiento de la persona viviendo en su domicilio y el apoyo al cuidador familiar (Jeon, 2004) y parecen facilitar la adaptación de los cuidadores al proceso del cuidado (Gottlieb y Johnson, 2000). En estos centros, las personas con demencia acuden un número específico de horas semanales, reciben los cuidados necesarios y realizan diferentes actividades en función de su nivel cognitivo y funcional. Sin embargo, aunque parezca contradicto- rio, Gottlieb y Johnson (2000) encontraron en la literatura numerosos ejemplos en los que el porcentaje de ingresos residenciales es mayor en aquellas personas que hicieron uso de los centros de día que en aquellas que no lo hicieron, por lo que el uso de centros de día parece ser un paso intermedio al ingreso en lugar de servir como un programa exclusivamente de respiro para los cuidadores informales. Esto mismo se ha puesto de manifiesto en una reciente investigación en nuestro contexto sociocultural (López, Losada, Romero-Moreno, Márquez-González y Martínez-Martín, 2012).

Pese a que algunas investigaciones deducen que la asistencia a centros de día parece tener efectos beneficiosos en sí mismos, como disminuir el estrés del cuidador o reducir la frecuencia de conductas disruptivas de los familiares con demencia los días que han hecho uso del servicio (Zarit, Kim, Femia, Almeida, Savla y Molenaar, 2011), los resultados sobre la eficacia de los centros de día para reducir el malestar en el cuidador se han mostrado inconsistentes (Gottllieb y Johnson, 2000). Además, a pesar de que los cuidadores expresan alto grado de satisfacción con el uso de los centros, los cuidadores (especialmente mujeres) con puntuaciones altas en depresión o sobrecarga informan de problemas y dificultades para usar este servicio asistencial (Jarrott, Zarit, Parris-Stephens, Townsend y Greene, 1999). Parece que para trabajar con el malestar del cuidador es necesario un apoyo adicional aparte del uso de los centros de día (Zarit, Gaugler y Jarrott,1999). Una posible solución sería combinar el uso del centro de día con otro tipo de intervenciones, especialmente programas psicoeducativos para el entrenamiento en manejo de estrategias y habilidades para afrontar el 
cuidado y para cubrir las necesidades de los cuidadores principales, disminuyendo así sus niveles de malestar. Sería especialmente recomendable que estas intervenciones se ofrecieran desde los propios centros de día por la mayor facilidad para acceder a la población de cuidadores, la familiaridad con los problemas que se pueden presentar y el mayor conocimiento de las problemáticas concretas de cada persona mayor usuaria de los mismos. Las intervenciones psicosociales, como el apoyo y la educación al cuidador, la modificación ambiental y el consejo, pueden retrasar la necesidad de institucionalización y pueden disminuir la carga que supone el cuidar a personas con demencia (Logsdon et al., 2007).

Uno de los obstáculos que parecen consolidar el hecho de que muchas familias no utilicen los centros tiene también que ver con los problemas de conducta del familiar. Así, los comportamientos problemáticos (agitación, agresividad, tristeza...) son una de las dificultades más frecuentemente señaladas en la literatura científica sobre personas con Alzheimer u otras demencias y están recibiendo una gran atención en la investigación (Algase, Beck, Kolanowski, Whall, Berent, Richards y Beattie, 1996; Cohen-Mansfield y Mintzer, 2005; Losada, 2004) y se han asociado con un menor uso de los servicios de respiro (Mavall y Malmberg, 2007). Además de ser uno de los factores que más afecta a la carga del cuidador, requieren un gran despliegue de recursos tanto individuales como institucionales (Wimo, Wimbland y Jönsson, 2010) y son considerados una de las mayores causas de institucionalización (Hamel, Gold, Andres, Reis, Dastoor, Grauer y Bergman, 1990; Mittelman, Roth, Haley y Zarit, 2004; Cohen- Mansfield y Mintzer, 2005).
De entre todas las situaciones del cuidado en las que pueden aparecer estos problemas de conducta, la situación de prepararse para acudir al centro de día es un momento especialmente sensible para su aparición (Arksey, Jackson, Croucher, Weatherly, Golder, Hare, Newbronner y Baldwin, 2004; Gottlieb y Johnson, 2000). Pese a que el centro de día está pensado como un servicio de respiro, es frecuente que los cuidadores consideren que emplean más tiempo tratando de preparar a su familiar para ir que el que emplean cuando no van. De hecho, preparar al familiar para que asista al centro de día y, en algunos casos, transportar al familiar al centro y volver del centro, es una importante barrera para el uso de los centros de día (Gaugler, Kane, Kane, y Newcomer, 2005). Además, algunos cuidadores informan de que el ir y venir al centro añade confusión y malestar a sus familiares (Gottllieb y Johnson, 2000) y que el tiempo libre que obtienen por el respiro lo dedican a "ponerse al día" en otras tareas y no a su cuidado personal y disfrute, provocando su uso más estrés que respiro (Worcester y Hedrick, 1997).

Se están obteniendo resultados prometedores para la intervención sobre problemas de conducta en personas con demencia a través de las denominadas terapias no farmacológicas (Cohen-Mansfield, 2001; Cohen-Mansfield y Mintzer, 2005; Logsdon et al., 2007; Losada, 2004; Olazarán, Reisberg, Clare, Cruz, Peña-Casanova, Del Ser, Woods, Beck, Auer, Lai, Spector, Fazio, Bond, Kivipelto, Brodaty, Rojo, Collins, Teri, Mittelman, Orrell, Feldman y Muñiz, 2010). Las terapias en las que se utilizan técnicas derivadas de la modificación de conducta (por ej., Bourgeois, Burgio, Schulz, Beach y Palmer, 1997) han recibido suficiente respaldo en diferentes 
estudios como para ser consideradas empíricamente validadas (Logsdon et al., 2007).

Partiendo de todo lo anterior, en el equipo de investigación "Cuidemos" se ha llevado a cabo una investigación piloto (NogalesGonzález, Losada, Márquez-González y Zarit, enviado) que tenía como objetivo entrenar a cuidadores en técnicas de modificación de conducta para reducir los problemas de conducta relacionados con la resistencia a acudir al centro de día, a través de intervenciones individuales de 6 sesiones. Además de evaluar los problemas de conducta, se evaluaron otras dimensiones psicológicas del cuidador (depresión y ansiedad), para analizar los posibles efectos indirectos de la intervención sobre el malestar de los cuidadores. La primera conclusión a la que se llegó con este estudio piloto es que un porcentaje significativo de las personas que acuden como usuarios a un centro de día y que están diagnosticadas de demencia presentan problemas de conducta relacionados con la asistencia al centro de día $(62,8 \%)$. Se observaron reducciones significativas en las conductas disruptivas que se habían propuesto como objetivo en los tres participantes en el estudio. Además, el programa resultó efectivo para reducir de forma clínicamente significativa la sintomatología depresiva y la ansiedad en dos de los tres cuidadores. Los resultados de este estudio sugieren que la intervención individual puede resultar útil para modificar los problemas de conducta de la persona con demencia relacionados con la asistencia al centro de día.

\section{4 - Atención a familias en residencias}

Otro escenario de acción fundamental de las personas que cuidan y que ha sido poco analizado es el contexto de las residencias o unidades de larga estancia en las que residen sus familiares. En contra de la idea bastante generalizada de que ingresar a una persona mayor en una residencia implica un abandono por parte de sus familiares, es evidente que una gran cantidad de cuidadores siguen implicados en el cuidado de sus familiares tras la institucionalización (Chen, Sabir, Zimmerman, Suitor y Pillemer, 2007; Davis y Buckwalter, 2001). De este modo, el rol de cuidador rara vez desaparece completamente una vez tomada la difícil decisión de institucionalizar al familiar, si bien sí parece cambiar de forma sustancial a partir de este momento.

Como se ha sugerido, tomar la decisión del ingreso suele ser difícil para los cuidadores, y todo el proceso de transición que implica la institucionalización suele implicar costes emocionales importantes para ellos. Son frecuentes en este proceso emociones como la tristeza, la frustración, los miedos, la incertidumbre sobre cómo será tratado su familiar, la desorientación y experiencias emocionales más complejas como la mezcla de alivio y culpabilidad, entre otras.

A lo largo de todo el proceso de la institucionalización, el apoyo y consejo psicológico ha demostrado ser beneficioso para el bienestar emocional de estas personas. Así, Gaugler, Roth, Haley y Mittelman (2008) aplicaron un programa de atención psicológica consistente en 6 sesiones de asesoría individual y familiar combinada con participación en un grupo de apoyo mutuo y consultoría telefónica disponible en cualquier momento y consiguieron reducir el nivel de carga y depresión de los cuidadores en el momento de la institucionalización y tras ésta. Por otra parte, tener relaciones satisfactorias y positivas con el 
personal de la residencia también facilita a los cuidadores el afrontamiento del estrés emocional causado por el proceso de institucionalización (Garity, 2006).

Una vez que transcurre la fase de transición que supone la institucionalización, los familiares deben seguir siendo parte activa del equipo de cuidados. De hecho, facilitar y potenciar, desde las instituciones, la implicación de los familiares en el cuidado de la persona mayor institucionalizada es un objetivo cuya importancia ha sido subrayada en múltiples foros y avalada por diversos estudios. La mayor implicación de la familia en el cuidado del residente se ha encontrado asociada con mayor bienestar físico y psicológico de éste (p.ej., McCallion, Toseland y Freeman, 1999). La familia constituye el núcleo principal de las relaciones sociales y afectivas de la persona mayor y su presencia activa en la residencia garantiza el que ésta disfrute de interacciones emocionales significativas, cercanas e íntimas, al tiempo que potencia su orientación e identidad, al facilitar la percepción de continuidad para el residente. Este papel de mantenedores de la identidad parece ser especialmente importante cuando el familiar institucionalizado presenta deterioro cognitivo o demencia. Una mayor integración de las familias en las residencias también se asocia con una mayor satisfacción y bienestar emocional (menor culpa y carga) en los propios cuidadores (Gaugler, Anderson, Zarit y Pearlin, 2004; Tilly y Reed, 2006), muchos de los cuales encuentran gratificante poder seguir implicados en el cuidado del familiar. También el personal del centro parece informar de mayor satisfacción cuando hay una mayor integración de los familiares en éstos (Robison y Pillemer, 2005; Pillemer, Sui- tor, Henderson, Meador, Schultz, Robison y Hegeman, 2003).

Sin embargo, existen bastantes obstáculos para lograr esta deseada integración familia-residencia. Por parte de los familiares, estereotipos sobre las residencias, falta de información y claridad (ambigüedad) sobre su papel como familiares en el cuidado del residente, dificultades de comunicación con el personal o emociones difíciles como la ansiedad o la culpa que sienten cuando visitan a sus familiares pueden ser algunos de los más destacables. Por parte de los centros, estos no parecen ofrecer a los familiares cauces formales explícitos para participar en el cuidado, lo que se asocia con la ausencia de una filosofía de centro que explicite y asuma como objetivo fundamental del mismo la atención e integración de los familiares en el cuidado del residente. De este modo, en la práctica, la familia no suele encontrar huecos reales en los que poder ejercer su colaboración en el cuidado. Por su parte, el personal de la residencia se encuentra, a su vez, sobrecargado con sus múltiples tareas prioritarias centradas en el cuidado técnico y de los residentes, mostrando una excesiva rutinización de su trabajo y no disponiendo de tiempo para dedicarlo a los familiares.

Estas circunstancias facilitan en muchas ocasiones la aparición de tensiones y dificultades en las relaciones entre el personal y los familiares, quienes, por un lado, suelen desear que el personal trate a sus familiares de forma más individualizada e integral (menos "técnica") y, por otro lado, se sienten ignorados y no reconocidos por los profesionales del centro.

Se han señalado diferentes medidas que pueden contribuir a superar estos obstáculos y favorecer la colaboración entre la 
familia y el personal del centro, llegando a proponerse intervenciones estructuradas para lograr este objetivo. La intervención más popular en este sentido es el programa "Compañeros en el Cuidado" desarrollada por el investigador Karl Pillemer y colaboradores (Pillemer et al., 2003; Robison y Pillemer, 2005). Este programa consiste en dos talleres paralelos de 7 horas de duración, uno impartido a los familiares y el otro al personal de enfermería del centro residencial, incluyendo una sesión final conjunta en la que familiares y profesionales se comunican para intentar mejorar sus relaciones y tomar decisiones que faciliten su colaboración en el cuidado. Los contenidos fundamentales del programa son el entrenamiento en habilidades de comunicación como la escucha activa, la empatía, el manejo de conflictos, la consideración de valores y las diferencias culturales y la discusión abierta sobre sus sentimientos y pensamientos de un grupo hacia el otro. A través de esta intervención se han obtenido efectos beneficiosos tanto para los familiares (quienes perciben una mayor empatía por parte de los profesionales e informan de un menor nivel de depresión tras la intervención), como para los profesionales de los centros, que perciben a los familiares como más colaboradores y presentan menor intención de abandonar su puesto de trabajo (Pillemeret al., 2003). El programa Compañeros en el Cuidado todavía no ha sido replicado en nuestro país, aun cuando ésta, y otras intervenciones, podrían contribuir enormemente a mejorar la calidad de vida de las familias que continúan cuidando a sus familiares en las residencias. Es innegable el hecho de que en numerosos centros residenciales de nuestro país se han desarrollado y se llevan a cabo iniciativas dirigidas a facilitar la colaboración de la familia en la residencia, si bien todavía es escasa la sistematización de estos procedimientos y su difusión a través de publicaciones.

Como se desprende de lo revisado en esta sección, es indudable que las personas que cuidan pueden seguir siendo cuidadoras tras la institucionalización de su familiar, si bien su rol cambia de forma sustancial, presentando nuevas necesidades y dificultades. Desde los centros residenciales, se debería atender de forma satisfactoria a las familias para ayudarles en este proceso, desde los primeros momentos de la interacción con éstas.

\section{5- Cuidadores en el duelo. El contexto en el que el familiar ya no está}

El duelo ante la muerte de un ser querido con demencia, como cualquier otra experiencia dolorosa, es parte de la vida. Sin embargo, cuando fallece un ser querido, la sociedad "exige" dos posturas que pueden resultar contradictorias (López y Quintero, 2010); por una parte, que se manifieste un cierto pesar ante la pérdida de un ser querido. No parece normal encontrarse contento y feliz, ni siquiera tranquilo, en esos momentos inmediatamente posteriores a que fallezca un familiar. Después de saber que ha fallecido un ser querido "se tiene" que estar triste y acongojado. Entre los cuidadores de familiares con demencia hay algunos de ellos que se sienten culpables por no sentirse mal después del fallecimiento del familiar al que cuidaban o, más exactamente, por no sentirse tan mal como habían previsto que sucedería. Es decir, hay cuidadores que se sienten mal por no sentirse mal cuando fallece un ser querido.

La segunda solicitud es que no se esté mucho tiempo afligido por la ausencia del ser 
querido que falta. El duelo, para ser socialmente aceptable, tiene que ser relativamente fugaz. Parece que el duelo es una reacción normal ante la pérdida de una persona amada, que es una respuesta adaptativa, siempre que no dure demasiado. Tanto es así que los criterios recogidos en el DSMIV-TR, pese a reconocer explícitamente que "la duración y la expresión de un duelo «normal» varía considerablemente entre los diferentes grupos culturales", indican que por encima de dos meses puede diagnosticarse un trastorno depresivo si se mantienen, además de sentimientos intensos de tristeza, algunos síntomas asociados como insomnio, anorexia y pérdida de peso, y no se debe diagnosticar un duelo complicado antes de transcurridos seis meses de la pérdida. Aunque, evidentemente, para tratar de aliviar el sufrimiento de quien ha perdido a un ser querido no "hace falta" esperar seis meses.

Algunos autores (Holland, Currier y Gallagher-Thompson, 2009; Silverberg, 2006; Walker, Pomeroy, McNeil y Franklin, , 1994) indican que en el caso de los cuidadores de personas con demencia se puede dar un duelo privado de manifestación, un duelo que no siempre es bien acogido por los demás, ya que consideran el fallecimiento un alivio más que una causa de duelo.

Cada cual tiene su propia manera de adaptarse a la ausencia de sus seres queridos. El duelo es un proceso personal caracterizado por la idiosincrasia, intimidad y peculiaridad de nuestra identidad (Neimeyer, 2002; Silverberg, 2006). El duelo es una experiencia a la vez universal y única, siendo patológico cuando interfiere sensiblemente en el funcionamiento general de la persona, comprometiendo su salud, bien sea poco después del fallecimiento del ser querido, bien transcurrido un gran periodo de tiempo desde su muerte.

El proceso de duelo se manifiesta en diferentes etapas descritas con pequeñas variaciones por diversos autores. Neimeyer (2002) considera que el doliente pasa de la evitación a la asimilación, para terminar con la acomodación, mientras que KüblerRoss (1989) señala que las fases del duelo en los pacientes terminales son: negación, depresión, cólera, reajuste y aceptación.

Hay al menos tres teorías sobre el proceso de duelo en los cuidadores. Una primera teoría de la acumulación de estrés, dice que conforme aumenta el tiempo y estresores del cuidado el duelo es peor. Cuanto más tiempo haga que se es cuidador, el estrés aumenta, los problemas son mayores, los recursos del cuidador son menores y, por tanto, el proceso del duelo se complica. Una segunda teoría, de la reducción de estrés, dice justamente lo contrario, pues se señala que cuanto mayor es el tiempo cuidado menor es el impacto del duelo. Cuanto más tiempo haga que se es cuidador el estrés disminuye pues los cuidadores se adaptan mejor a los problemas que van surgiendo y en este mismo sentido el proceso del duelo se aborda mejor. Una última teoría señala que gran parte del duelo se elabora anticipatoriamente en los cuidadores, facilitando el duelo al fallecer la persona con demencia. El cuidador que afronta una enfermedad como la demencia usualmente comienza el proceso de duelo antes de la pérdida propiamente dicha. Las tareas a llevar a cabo en el duelo se realizan en parte antes de que el familiar fallezca y esto hace que el duelo cuando fallece el ser querido sea más fácil de llevar a cabo (Schulz et al., 2008). 
El duelo es el proceso de reconstruir el mundo (y por tanto, a sí mismo) sin la persona perdida. Se trata de dotar de un nuevo sentido a los elementos con los que el sujeto debe construir su realidad. Worden (2004) propone cuatro tareas para superar el duelo de forma satisfactoria. Lo que resulta más interesante del planteamiento de este autor es que otorga gran responsabilidad al individuo en su proceso de reconstruir su mundo sin la persona fallecida al establecer "tareas" y no "etapas" del duelo, por lo que da un papel activo frente a otras definiciones tradicionales menos participativas. El concepto de etapa es algo por lo que hay que pasar, mientras que el de tarea es algo que yo puedo hacer, un trabajo o reto que se pone delante de uno. A su juicio, son cuatro las tareas a llevar a cabo, que no necesariamente siguen el orden que se propone a continuación:

Aceptar la realidad de la pérdida. Por muy previsible que sea el fallecimiento de un familiar siempre hay una cierta sensación inicial de que no es real lo que ha pasado. La primera tarea es, pues, asumir que el ser querido no va a volver, que el reencuentro no es posible.

Trabajar las emociones y el dolor de la pérdida. El dolor que se siente es un dolor total: biológico, psicológico, social, familiar y espiritual. No siempre el dolor tiene la misma intensidad pero no se puede evitar el dolor de la pérdida y las sensaciones asociadas, generalmente, enfado, culpa, soledad, impotencia o tristeza.

Adaptarse a un medio en el que el fallecido ya no está. Esta tercera tarea es esencial ya que el proceso fundamental de la experiencia de duelo es el intento de reconstruir el propio mundo de significados.
Recolocar emocionalmente al fallecido $y$ continuar viviendo. Hay que encontrar un lugar apropiado en la vida emocional al ser querido que ha fallecido que deje espacio para los demás. No se trata de olvidar sino que el recuerdo del ser fallecido no le impida sentirse cómodo y volver a vivir la vida. Esta cuarta tarea termina cuando se percibe al mundo con sentido, con otros seres a los que amar, aunque ya no esté el ser querido.

Pasar de estar centrados en la muerte, en el morir (en lo que se ha perdido y los sentimientos dolorosos que se experimentan) a estar centrados en la vida, en el vivir (en lo que se es y se tiene, y en el ser capaces de amar), que cicatricen las heridas, aceptar intelectual y emocionalmente la pérdida, reconstruir la vida y su significado, no es un proceso ni corto, ni fácil (López y Quintero, 2010). Con todo, muchos de los cuidadores se muestran resilientes ante el duelo (Holland et al., 2009). De todos los cuidadores de familiares con demencia en duelo una tercera parte utiliza algún recurso en esos momentos, bien consejo, psicofármacos o bien un grupo de ayuda mutua. Son los cuidadores emocionalmente más afectados los que más utilizan estos recursos (Bergman, Haley y Small, 2011).

Es esencial que, en la medida de lo posible (López y Quintero, 2010; Walker et al., 1994), el entorno de la persona en duelo se comprometa, pues los familiares, amigos y vecinos son una ayuda relevante en este proceso. También es muy importante facilitar la comunicación. Así, si el cuidador desea hablar del fallecido, o de sus propios sentimientos, conviene facilitarlo. Es positivo dar información pero que ésta se ajuste a la capacidad de asimilación y uso del cuidador. Asimismo, conviene ser flexibles: hay muchas diferencias no sólo entre 
las distintas personas que pierden a un mismo ser querido sino también en una misma persona a lo largo del tiempo, no siendo lo mismo perder a un familiar mayor que a un esposo de mediana edad o un hijo. Conviene adaptarse a las distintas situaciones, la muerte siempre es algo nuevo. También es importante respetar la expresión de sentimientos del cuidador; estos sentimientos pueden variar desde la tristeza hasta el enfado y la rabia. No siempre es fácil acompañar la rabia contra los otros, contra el mundo o contra Dios.

Aunque el apoyo empírico sobre la eficacia de los tratamientos para mejorar la adaptación al duelo es inconsistente, parece que las intervenciones dirigidas a tratar el estrés y la depresión de los cuidadores consiguen disminuir el riesgo de duelo complicado después del fallecimiento del familiar con demencia. Estos resultados sugieren que los tratamientos dirigidos a prevenir el duelo a través del control del estrés del cuidador pueden ser eficaces. Cuidadores que tras un proceso terapéutico saben manejar el estrés y los sentimientos depresivos que les acontecen parecen que se adaptan mejor a los efectos de la pérdida del familiar, que son más capaces de afrontar el duelo cuando fallece su ser querido (Schulz, Boerner y Hebert, 2008; Holland et al., 2009).

Para acompañar el duelo normal, y con el fin de que no se complique ni se enmascare, ni se cronifique y luego explote, conviene que una vez que el familiar ha superado el estado inicial de shock por la noticia del fallecimiento del ser querido, se hable con él para facilitar, o por lo menos no entorpecer, las tareas que ayudan a la recuperación del duelo (Walker et al., 1994). Según Silverberg (2006), tras fallecer el familiar con demencia, la mayoría de los cuidado- res no reconocen su duelo y por tanto una primera tarea para el terapeuta será trabajar el reconocimiento. Luego, habrá que ayudar al doliente a que acepte la realidad de la pérdida, trabaje las emociones y el dolor de la pérdida, se adapte a un medio en el que el fallecido está ausente y recoloque emocionalmente al fallecido para poder seguir viviendo.

Kasl-Godley (2003), en un estudio piloto para ayudar a cuidadores de familiares con demencia a manejar las reacciones de duelo, encuentra que los cuidadores estaban satisfechos al terminar el tratamiento y que habían normalizado su experiencia de duelo.

Burns, Nichols, Martindale-Adams, Graney y Lummus (2003) comparan dos intervenciones dirigidas a paliar el duelo de cuidadores con demencia. Los autores consiguen reducir significativamente el malestar provocado por los problemas de memoria y de conducta y aumentar el bienestar subjetivo.

La mayoría de los cuidadores necesitan apoyo un breve espacio de tiempo, algunos necesitan de acompañamiento durante un tiempo más prolongado y sólo unos pocos necesitarán la ayuda de profesionales de la salud mental (Holland et al., 2009). Con todo, y pese a estas diferencias entre las personas, hay que tratar de dar a todos la ayuda que precisan.

\section{6- Conclusiones}

Son muchas las familias y personas que individualmente asumen la mayor parte de los cuidados de familiares enfermos con demencia sufriendo importantes consecuencias negativas sobre su salud psicológica y física asociadas al cuidado del 
familiar. A través del presente trabajo se ha pretendido reflejar que, a pesar de que la mayoría de ejemplos de estudios sobre intervenciones dirigidas a cuidadores familiares de personas con demencia se llevan a cabo cuando el cuidado se está realizando en el domicilio, la realidad es que los cuidadores continúan realizando su labor en otros contextos. A pesar de que determinados servicios como los centros de día o las residencias puedan, entre otras cuestiones, proporcionar al cuidador un cierto respiro en sus tareas, aún pueden realizarse importantes actuaciones que beneficien más a las familias. Por ejemplo, se pueden realizar intervenciones que actúen sobre la sorprendente infrautilización de recursos formales de respiro. Si se analizan con detalle los factores, relacionados con el cuidado, que están tras la no utilización de los mismos, se pueden encontrar importantes dimensiones sobre la que se puede intervenir para aumentar su uso (p.ej., intervenciones conductuales para evitar comportamientos problemáticos). Complementar la ayuda formal con recursos específicos de ayuda al cuidador puede contribuir de manera significativa a mejorar la calidad de vida de los cuidadores y de sus familias, así como retrasar o incluso evitar (si así lo desea la familia) la institucionalización de la persona que padece demencia.

Por otra parte, se ha pretendido reflejar, de forma contraria a la creencia popular de que el cuidado termina cuando se ingresa al familiar en una residencia o éste fallece, que se puede hacer mucho por disminuir el sufrimiento de los cuidadores en etapas tan difíciles como las de la institucionalización o el fallecimiento del familiar.

Si bien cada vez resulta más habitual encontrar ejemplos de investigaciones diri- gidas a mejorar la calidad de vida de los cuidadores en el contexto domiciliario (ver, por ejemplo, Etxeberría et al., 2011; López et al., 2007; Losada et al., 2004, 2011; Márquez-González et al., 2007, 2010; MartínCarrasco et al., 2009), la eficacia de las mismas es aún moderada (Pinquart y Sörensen, 2006), y el número de iniciativas realizadas en nuestro país es extremadamente escaso. Se han revisado diferentes propuestas de intervenciones para ayudar a los cuidadores (ver, p. ej., Gallagher-Thompson et al., 2012), y se ha sugerido la necesidad de contar con iniciativas que analicen cómo ayudar a los cuidadores en otros contextos o etapas del cuidado. A pesar de que el contexto actual de crisis parece limitar aun más las posibilidades de ofrecer una adecuada ayuda a las familias que cuidan, la literatura científica parece reflejar cada vez más iniciativas y procedimientos que pueden contribuir a proporcionar una ayuda eficaz a las familias. Tal y como se ha reflejado en este artículo, existen diferentes opciones no farmacológicas que pueden ayudar tanto a los cuidadores como a las personas que padecen demencia.

Addis, M.E. y Krasnow, A.D. (2000). A national survey of practicing psychologists' attitudes toward psychotherapy treatment manuals. Journal of Consulting and Clinical Psychology, 68, 331-339.

Algase, D.L, Beck, C., Kolanowski, A., Whall, A., Berent, S., Richards, K. y Beattie, E. (1996). Need-Driven dementia-compromised behavior: An alternative view of disruptive behavior. American Journal of Alzheimer's Disease, 11, 10-19.

Arksey, H., Jackson, K., Croucher, K., Weatherly, H., Golder, S., Hare, P., Newbronner, E. y Baldwin, S. (2004). Review of respite services and short-term breaks for carers for people with dementia. Report for the National 
Co-ordinating Center for NHS Service Delivery and Organisation $R \&$ \& (NCCSDO), July 2004.

Bergman, E. J., Haley, W. E., y Small, B. J. (2011). Who uses bereavement services? An examination of service use by bereaved dementia caregivers. Aging and Mental Health, 15, 531-540.

Bourgeois, M.S., Burgio, L.D., Schulz, R., Beach, S. y Palmer, B. (1997). Modifying Repetitive Verbalizationsof Community-Dwelling Patients with AD. The Gerontologist, 37, 30-39.

Burgio, L., Lichstein, K.L., Nichols, L., Czaja, S., Gallagher-Thompson, D., Bourgeois, M., Stevens, A., Ory, M., Schulz, R. y REACH Investigators. (2001). Judging Outcomes in Psychological Interventions for Dementia Caregivers: The Problem of Treatmentlmplementation. The Gerontologist, 41, 481-489.

Burns, R., Nichols, L. 0., Martindale-Adams, J., Graney, M. J., y Lummus, A. (2003). Primary care interventions for dementia caregivers: 2-year outcomes from the ReachStudy. The Gerontologist, 43, 547-555.

Chen, C.K., Sabir, M., Zimmerman, S., Suitor, J., y Pillemer, K. (2007). The importance of family relationships with nursing facility staff for family caregiver burden and depression. Journals of Gerontology, 62, 253-260.

Cohen-Mansfield, J. y Mintzer, J.E. (2005). Time for change: The role of nonpharmacological interventions in treating behavior problems in nursing home residents with dementia. AlzehimerDissabilities Association Disorder, $19,37-40$.

Cohen-Mansfield, J. (2001). Nonpharmacologic Interventions for Inappropriate Behaviors in Dementia.A Review, Summary and Critique. American Journal of Geriatric Psychiatry, 9, 361-381.

Crespo M., López, J., y Zarit, S.H. (2005). Depression and anxiety in primary caregivers: a comparative study of caregivers of demented and nondemented older persons. International Journal of Geriatric Psychiatry.20, 591-592.

Davis, L. y Buckwalter, K. (2001). Family caregiving after nursing home admission. Journal of Mental Health and Aging, 7, 361-379.

Etxeberria, I., García-Soler, A., Iglesias, A., Urdaneta, E., Lorea, I., Díaz, P. y Yanguas, J.J. (2011). Effects oftraining in emotional regulation strategies on the well-being of carers of Alzheimer patients. Revista Española de Geriatría y Gerontología, 46, 206-212.

Gallagher-Thompson, D. y Coon, D. (2007). Evidence-based psychological treatments for distress infamily caregivers of older adults. Psychology and Aging, 22, 37-51.

Gallagher-Thompson, D., Tzuang, M.Y., Brodaty, H., Charlesworth, G., Gupta, R., Lee, S.E., Losada, A. yShyu, Y.I.. (2012). International Perspectives on Nonpharmacolo- gical Best practices for Dementia Family Caregivers: A Review. Clinical Gerontologist, 35, 316-355.

Garity J. (2006). Caring for a family member with Alzheimer's disease: coping with caregiver burden postnursing home placement. Journal of Gerontological Nursing, 32, 39-48.

Gaugler, J.E., Anderson, K.A., Zarit, S.H. y Pearlin, L.I. Family involvement in nursing homes: effects on stress and well-being. Aging and Mental Health, 8, 65-75.

Gaugler, J., Kaen, R., Kane, R. y Newcomer, R. (2005). Early Community-Based Service Utilization and its Effects on Institutionalization in Dementia Caregiving. The Gerontologist, 45, 177-185.

Gaugler, J.E., Roth, D.L., Haley, W.E., y Mittelman, M.S. (2008). Can counseling and support reduce burden and depressive symptoms in caregivers of people with Alzheimer's disease during the transition to institutionalization? Results from the New York University caregiver intervention study. Journal of the American Geriatrics Society, 56, 421-428

Gottlieb y Johnson. (2000). Respite programs for caregivers of persons with dementia: A review with practice implications. Aging and Mental Health, 4, 119-129

Grant, I., Adler, K. A., Patterson, T. L.,Dimsdale, J. E., Ziegler, M. G. y Irwin, M. R. (2002). Healthconsequences of Alzheimer'scaregiving transitions: Effectsof placement and bereavement. Psychosomatic Medicine, 64,477-486.

Hamel, M., Gold, D.P., Andres, D., Reis, M., Dastoor, D., Grauer, H. y Bergman, H. (1990). Predictors and consequences of aggressive behavior by community-based dementia patients. The Gerontologist, 30, 206-211.

Holland, J. M., Currier, J. M. y Gallagher-Thompson, D. (2009). Outcomes From the Resources for Enhancing Alzheimer's Caregiver Health (REACH) Program for $\mathrm{Be}$ reaved Caregivers. Psychology and Aging, 24, 190-202.

IMSERSO (2005). Cuidado a las personas mayores en los hogares españoles. Madrid: IMSERSO.

IMSERSO (2008). Las personas mayores en España. Madrid: IMSERSO.

Jarrott, S.E., Zarit, S. H., Parris-Stephens, M. A., Townsend, A. y Greene, R. (1999). Caregiver satisfaction with adult day service programs. American Journal of Alzheimer's Desease, 14, 233-244

Jeon, Y.H. (2004). Shaping mutuality:Nurse-family caregiver interactions in caring for older people with depression. International Journal of Mental Health Nursing, 13, 126-134

Kasl-Godley, J. (2003). Anticipatory grief and loss: Implications for intervention. En D. Coon, D. GallagherThompson, y L. Thompson (Eds.), Innovative interventions 
to reduce dementia caregiver distress (pp. 210-219). New York: Springer.

Knight, B., y Losada, A. (2011). Family Caregiving for Cognitively or Physically Frail Older Adults: Theory, Research, and Practice. En K.W. Schaie y S.L. Willis (Eds.), Handbook of the Psychology of Aging (7a ed., pp. 353365). New York: Academic Press.

Knight, B. G. ySayegh, P. (2010). Cultural values andcaregiving: the updated Sociocultural Stress and CopingModel. Journals of Gerontology, 65, 5-13.

Kübler-Ross, E. (1989). Sobre la muerte y los moribundos. Barcelona: Grijalbo.

Larson, E.B., Shadlen, M.F., Wang, L., McCormick, W.C., Bowen, J.D., Teri, L. yKukull, W.A.(2004). Survival after initial diagnosis of Alzheimer disease. Annals of Internal Medicine, 140, 501-509.

Lieberrnan, M. A., y Fisher, L. (2001). The effects of nursinghome placement on familycaregivers of patients withAlzheimer's disease. The Gerontologist, 41, 819-826.

Logsdon, R.G., McCurry, S.M. y Teri, L. (2007). EvidenceBased Psychological Treatments for Disruptive Behaviors in Individuals with Dementia. Psychology and Aging, 22, 28-36.

López, J., Crespo, M. y Zarit, S. H. (2007). Assessment of the efficacy of a stress management programfor informal caregivers of dependent older adults. The Gerontologist, 47, 205-214.

López, J. y Quintero, A. (2010). Acompañar a las familias en el duelo de un ser querido. ITER, 51, 155-174.

López, J., Losada, A., Romero-Moreno, R., MárquezGonzález, M., y Martínez-Martín, P. (2012). Factores asociados a la consideración de ingresar a un familiar con demencia en una residencia. Neurología, 27, 83-89

Losada, A. (2004). Intervenciones no farmacológicas para la reducción de comportamientos problemáticos asociados a la demencia. Madrid: Portal Mayores.

Losada, A., Izal, M., Montorio, I., Márquez, M., y Pérez, G. (2004). Eficacia diferencial de dos intervenciones psicoeducativas para cuidadores de familiares con demencia. Revista de Neurología, 38, 701-708.

Losada, A., Knight, B.G. y Márquez, M. (2003). Barreras cognitivas para el cuidado de personas mayoresdependientes. Influencia de las variables socioculturales. $R e$ vista Española de Geriatría y Gerontología, 38, 116-123.

Losada, A., Márquez-González, M., Knight, B.G., Yanguas, Y., Sayegh, P. y Romero-Moreno, R. (2010). Psychosocialfactors and caregivers' distress: effects of familism and dysfunctionalthoughts. Aging and Mental Health, 14, 193-202.
Losada, A., Márquez-González, M., Peñacoba, C., Gallagher-Thompson, D. y Knight B.G. (2007). Reflexiones en torno a la atención a los cuidadores informales de personas con demencia y propuesta de una intervención interdisciplinar. Psicología conductual,15, 57-76.

Losada, A., Márquez-González, M. y Romero-Moreno, R. (2011). Mechanisms of action of apsychological intervention for dementia caregivers: effects of behavioural activation andmodification of dysfunctional thoughts. International Journal of Geriatric Psychiatry, 26, 1119-1127.

Losada, A., Montorio, I., Knight, B.G., Márquez, M. e Izal, M. (2006). Explanation of caregivers distress from the cognitive model: the role of dysfunctional thoughts. Psicología Conductual, 14, 115-128.

Márquez-González, M., Losada, A., Izal, M., Pérez-Rojo, G., y Montorio, I. (2007). Modification of Dysfunctional Thoughts about Caregiving in Dementia Family Caregivers: description andoutcomes of an Intervention Program. Agingand Mental Health, 11, 616-625.

Márquez-González, M., Losada, A., Pillemer, K., RomeroMoreno, R., López, J. y Martínez, T. (2010). Cuidando más allá del domicilio: el papel de la familia en los centros residenciales y el cuidado colaborativo. Psicogeriatría, 2, 93-104.

Márquez-González, M., Romero-Moreno, R. y Losada, A. (2010). Caregiving issues in a therapeutic context: New insights from the acceptance and commitment therapy approach. En N. Pachana, K. Laidlaw y Bob Knight (Eds.). Casebook of Clinical Geropsychology: International Perspectives on Practice (pp. 33-53). New York: Oxford. University Press.

Martín-Carrasco, M., Martín, M.F., Valero, C.P., Millán, P.R., García, C.I., Montalbán, S.R., Vázquez, A.L., Piris, S.P. y Vilanova, M.B...(2009). Effectiveness of a psychoeducational intervention program in the reduction of caregiver burdenin Alzhimer's disease patients' caregivers. International Journal of Geriatric Psychiatry, 24, 489-499.

Mateos, R., Franco, M. y Sánchez, M. (2010). Care for dementia in Spain: the need for a nationwide strategy. International Journal of Geriatric Psychiatry, 25, 881-884.

Mausbach, B.T., Roepke, S.K., Depp, C.A., Moore, R., Patterson, T.L. y Grant, I. (2011). Integration of the pleasant events andactivity restriction models: development and validation of a "PEAR" model of negative outcomes in Alzheimer's caregivers. Behavior Therapy, 42, 78-88.

Mausbach, B.T., Roepke, S.K., Ziegler, M.G., Milic, M., von Känel, R., Dimsdale, J.E.,Mills, P.J., Patterson, T.L., Allison, M.A., Ancoli-Israel, S. y Grant I. (2010). Association between chronic caregiving stress and impaired endothelial function in the elderly. Journal of the American College of Cardiology, 8, 2599-2606. 
Mavall, L., y Malmberg, B. (2007). Day care for persons with dementia: An alternative for whom? The International Journal of Social Research and Practice,6, 27-43.

McCallion, P., Toseland, R.W., Freeman, K. (1999). An evaluation of a family visit education program. Journal of the American Geriatrics Society, 47, 203-214.

Mittelman, M., Roth, D., Clay, 0. y Haley, W. (2007). Preserving health of Alzheimer caregivers: Impact of a spouse caregiver intervention. American Journal of Geriatric Psychiatry, 15, 780-789.

Mittelman, M., Roth, D., Haley, W. y Zarit, S. (2004). Effects of a Caregiver Intervention on Negative Caregiving Appraisals of Behavior Problems in Patienswith Alzheimer's Desease: results of a Randomized Trial. Journal of Gerontology,59, 27-34.

Neimeyer, R. A. (2002). Aprender de la pérdida. Una guía para afrontar el duelo. Barcelona: Paidós.

Nogales-González, C., Losada, A., Márquez-González, M. \& Zarit, S.H. (submitted). Behavioral intervention for reducing resistance to attending day care centers: a pilot study.

Olazarán, J., Reisberg, B., Clare, L., Cruz, I., Peña-Casanova, J., Del Ser, T., Woods, B., Beck, C., Auer, S., Lai, C., Spector, A., Fazio, S., Bond, J., Kivipelto, M., Brodaty, H., Rojo, J.M., Collins, H., Teri, L., Mittelman, M., Orrell, M., Feldman, H.H. y Muñiz, R.(2010). Nonpharmacological Therapies in Alzheimer's Desease: A sistematic review of Efficacy. Dementia and Geriatric Cognitive Disorders,30, 161-178.

Pearlin, L. I., Mullan, J. T, Sernple, S. y Skaff, M. M. (1990). Caregiving and the stress process: An overview of concepts and theirmeasures. The Gerontologist, 30,583-591.

Pillemer, K., Suitor, J.J., Henderson,C.R.Jr, Meador, R., Schultz, L., Robison, J. y Hegeman, C. (2003). A cooperative communication intervention for nursing home staff and family members of residents. The Gerontologist, 43, 96-106.

Pillemer, K., Hegeman, C.R., Albright, B. y Henderson, C. (1998). Building bridges between families and nursing home staff: The partners in caregiving program. The $\mathrm{Ge}$ rontologist, 38, 499-503.

Pinquart, M., y Sörensen, S. (2003). Differences betweencaregivers and noncaregivers in psychological health andphysical health: A metaanalysis. Psychology and Aging, 18,250-267.

Pinquart, M., y Sörensen, S.(2006). Helping caregivers of persons with dementia: Which interventions work and how large are their effects? Intemational Psychogeriatrics, 18,577-595.

Pinquart M, y Sörensen S. (2011). Spouses, adult children, and children-in-law as caregivers of older adults: a meta-analytic comparison. Psychology and Aging, 26, 1-14.

Robison, J, y Pillemer, K. (2005). Partners in caregiving: Cooperative communication between families and nursing homes. In J. Gaugler (Ed.), Promoting family involvement in long-term care settings (pp. 201-224). Baltimore: Health Professions Press.

Robinson-Whelen, S., Tada, Y., MacCallum, R.C., McGuire, L. y Kiecolt-Glaser, J. K. (2001). Long term caregiving: What happenswhen it ends? Journal of Abnormal Psychology, 110, 573-584

Romero-Moreno, R., Losada, A., Mausbach, B.T., Márquez-González, M., Patterson, T.L. y López, J. (2011). Analysis of the moderating effect of self-efficacy domains in different points of the dementia caregiving process. Aging and Mental Health, 15, 221-231

Romero-Moreno, R., Márquez-González, M., Losada, A. y López J. (2011). Motives for caring: relationship to stress and coping dimensions. International Psychogeriatrics, $23,573-582$

Schulz, R. y Beach, S. R. (1999). Caregiving as a risk factor for mortality: The caregiver health effects study. JAMA, 282,2215-2219.

Schulz, R., Boerner, K, y Hebert, R. S. (2008). Caregiving and bereavement. En M. Stroebe, R. Hansson, H. Schut, y W. Stroebe, Handbook of bereavement research and practice (pp. 265-285). Washington: American Psychological Association.

Schulz, R., Belle, S. H., Czaja, S. J.,McGinnis, K. A., Stevens, A. y Zhang, S. (2004). Long-termcare placement of dementiapatients and caregiver healthand well-being. JAMA, 25,961-967.

Seligman, M.E.P. (1995). The effectiveness of psychotherapy: The Consumer Reports study. American Psychologist, 50, 965-974.

Silverberg, E. (2006). Introducing the 3-a grief intervention model for dementia caregivers: Acknowledge, assess and assist. Omega-Journal of Death and Dying, 54, 215-235.

Tilly J. y Reed P. (2006). Dementia care practice recommendations for assisted living residences and nursing homes. Washington: Alzheimer's Association.

Vitaliano, P.P., Young, H.M. y Zhang, J. (2004). Is caregiving a risk factor for illness?. Current Directions in Psychological Science, 13, 13-16.

Walker, R.J., Pomeroy, R.C., McNeil, J.S., y Franklin, C. (1994). Anticipatory grief and Alzheimer's disease: Strategies for interventions. Journal of Gerontological Social Work, 22, 21-23 
Wimo, A., Wimbland, B., y Jönsson, L. (2010). The worldwide societal costs of dementia: Estimates for 2009. Alzheimer's and Dementia, 6, 98-103.

Worcester M. y Hedrick S. (1997). Dilemmas in using respite for family caregivers of frail elders. Family and CommunityHealth, 19, 31-48.

Worden, J.W. (2004). El tratamiento del duelo: Asesoramiento psicológico y terapia ( $2^{\mathrm{a}} \mathrm{Ed}$.). Barcelona: Paidós.

Zarit, S.H. y Femia, E.E. (2008). A future for family care and dementia intervention research? Challenges and strategies. Aging and Mental Health, 12,5-13.

Zarit, S.H., Femia, E.E., Kim, K., y Whitlatch, C.J. (2010). The structure of risk factors and outcomes for family caregivers: implications for assessment and treatment. Aging and Mental Health, 14, 220-231.

Zarit, S.H., Gaugler, J.E. y Jarrott, S.E. (1999). Useful services for families: research findings and directions. International Journal of Geriatric Psychiatry. 14, 165-177.

Zarit, S. H., Kim, K., Femia, E. E., Almeida, D., Savla, J. y Molenaar, C. (2011). Effects of adult day careon daily stress of caregivers: a within- person approach. Journal of Gerontology, 66, 538-546.

Fecha de recepción: 20/07/2012

Fecha de aceptación: 10/02/2013 